Territorios 38 / Bogotá, 2018, pp. 95-117

ISSN: 0123-8418

ISSNe: 2215-7484

Dinámicas sociales y reconfiguraciones territoriales contemporáneas

\title{
El arrendamiento residencial en los barrios populares: las redes de relaciones y la generación de compromisos ${ }^{*}$
}

The Residential Lease in Popular Neighborhoods: The Network of Relations and the Generation of Compromises

O Arrendamento Residencial nos Bairros Populares: as Redes de Relações e a Geração de Compromissos

Hernando Sáenz Acosta ${ }^{* *}$

Recibido: 14 de agosto de 2017

Aprobado: 3 de octubre de 2017

Doi: http://dx.doi.org/10.12804/revistas.urosario.edu.co/territorios/a.5999

Para citar este artículo:

Sáenz, H. (2018). El arrendamiento residencial en los barrios populares: las redes de relaciones y la generación de compromisos. Territorios, (38), 95-117. Doi: http://dx.doi.org/10.12804/revistas.urosario.edu. $\mathrm{co} /$ territorios/a.5999

* La información aqui presentada recoge parcialmente los resultados de investigación de la tesis de doctorado titulada "A reciprocidade como marco institucional dos contratos de locação residencial: $O$ caso de Patio Bonito I, um bairro popular de Bogotá, na Colômbia”, que contó con el apoyo del programa de becas de posgrado PEC-PG CAPES Brasil entre los años 2011 y 2015. Se alimenta también de la ponencia "Por una lectura institucional beterodoxa de los contratos: El caso del mercado de vivienda en arrendamiento en un barrio de origen informal de Bogotá, Colombia", presentada en el III Seminario Internacional de la Red Latinoamericana de Investigadores sobre Teoría Urbana, que tuvo lugar en la ciudad de Querétaro, México, los días 11, 12 y 13 de octubre del 2016. El $\Longrightarrow$ 
Palabras clave

Análisis socioeconómico, convenciones, derecho a la vivienda, contratos, norma social, vivienda alquilada.

\section{Keywords}

Socioeconomic analysis, conventions, right to housing, contracts, social norm, rented housing.
Palavras-chave

Análise socioeconômico, convenções, Direito à vivenda, contratos, norma social, Vivenda alugada.

\section{territarios 38}

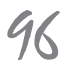

\section{RESUMEN}

El siguiente documento presenta un análisis del mercado de vivienda en arrendamiento en un barrio popular de la ciudad de Bogotá, y hace énfasis en los compromisos que se celebran entre arrendatarios y arrendadores. Para ello se presenta en un primer momento la metodología empleada en un trabajo de campo realizado entre el 2011 y el 2014 en el barrio Patio Bonito I, localizado al suroccidente de la ciudad. A partir de la Escuela de Convenciones Francesa como referente teórico, se exploran dos aspectos importantes para el funcionamiento de este mercado residencial: la existencia de redes de relaciones y la celebración de compromisos entre los agentes económicos. Estas características son cruciales para desarrollar una lectura institucional heterodoxa de este tipo de mercado - centrada en el concepto de las convenciones sociales- y comprender así las formas creadas por las comunidades en los barrios populares de la ciudad para su regulación.

\section{ABSTRACT}

The following document presents an analysis of the rental housing market in a popular neighborhood in the city of Bogotá, with emphasis on compromises between tenants and landlords. For this, the methodology used in a fieldwork carried out between 2011 and 2014 in the Patio Bonito I neighborhood, located in the southwest of the city is presented at first. Starting from the French School of Conventions as a theoretical reference, two important aspects for the functioning of this residential market are explored: the existence of networks of relations and the celebration of commitments among economic agents. These characteristics are crucial to develop a heterodox institutional reading of this type of market centered on the concept of social conventions and thus understand the forms created by communities in the city's popular neighborhoods for the regulation of this type of residential market.

\section{RESUMO}

O seguinte documento apresenta uma análise do mercado de vivenda em arrendamento em um bairro popular da cidade de Bogotá, fazendo ênfase nos compromissos que se celebram entre arrendatários e arrendadores. Para isto se apresenta em um primeiro momento a metodologia empregada em um trabalho de campo realizado entre 2011 e 2014 no bairro Patio Bonito I, localizado ao sul ocidente da cidade. Partindo da Escola de Convenções Francesa como referente teórico se exploram dois aspectos importantes para o funcionamento deste mercado residencial: a existência de redes de relações e a celebração de compromissos entre os agentes econômicos. Estas características são cruciais para desenvolver uma leitura institucional heterodoxa deste tipo de mercado centrada no conceito das convenções sociais e compreender assim as formas criadas pelas comunidades nos bairros populares da cidade para a regulação deste tipo de mercado residencial. 


\section{Introducción}

Uno de los principales medios para el acceso a la vivienda en Bogotá es el mercado de arrendamiento. Si bien las políticas actuales de vivienda están centradas en el acceso a la vivienda en propiedad, los datos censales muestran un incremento de la población que vive en arriendo: mientras que en 1993 este dato representaba un $41,6 \%$, en el 2005 era del $43 \%$ del total de hogares de la ciudad (Torres, 2012). Un dato más reciente que confirma el peso de esta forma de tenencia de vivienda lo proporciona la Encuesta Multipropósito 2014 donde se señala que la proporción de hogares que vive en arriendo en $\mathrm{Bo}^{-}$ gotá pasó del $41,4 \%$ al $46,8 \%$ entre los años 2011 y 2014 (Secretaría Distrital de Planeación, 2015, p. 56).

$\mathrm{El}$ acceso a la vivienda en arriendo es significativo entre las familias de menores ingresos de la ciudad. Al tomar como referencia el sistema que emplea la Alcaldía Mayor de Bogotá para la estratificación socioeconómica de la población, se puede observar que el peso de los arrendatarios es mayor en las zonas de la ciudad que presentan más deficiencias en materia urbanística ${ }^{1}$. Se trata de zonas clasificadas en estratos uno, dos y tres, donde el aumento de la población arrendataria ha sido similar o superior al promedio observado para la ciudad, en contraste con aquellas zonas que poseen una calidad urbanística superior: entre el 2011 y el 2014 la población arrendataria en estrato uno pasó de $41 \%$ a 47,9\%; en el estrato dos pasó de 47,7\% a $51,9 \%$, y en el estrato tres ascendieron de $42,2 \%$ a $47,3 \%^{2}$ (Secretaría Distrital de Planeación, 2015, p. 56).

$\mathrm{Si}$ bien existe una serie de trabajos destinados a la compresión del mercado de arrendamiento residencial en los barrios populares, hay un aspecto que ha sido poco explorado: la informalidad contractual presente allí ${ }^{3}$. Partiendo de la propuesta de Pedro Abramo (2009), se toma como referente para la distinción formal/informal, los aspectos jurídicos que envuelven las transacciones que se realizan en estos mercados habitacionales. La informalidad contractual ha sido considerada como una de las causas que explican la inseguridad en la tenencia de la vivienda por parte de los hogares arrendatarios. La inseguridad se define como aquellas situaciones en las cuales una persona o familia está bajo amenaza de desalojo de manera permanente, o en una situación ambigua por la cual alguna autoridad o agente privado puede cuestionar la posesión de la vivienda en cualquier momento. Entre 1998 y el 2008, cerca de 18 millones de personas en el mundo fueron víctimas de desalojo forzado; situación que ha sido considerada como crisis mundial en la inseguridad de la tenencia de vivienda (ONU, 2012).

Este artículo presenta a través de un análisis de caso algunas consideraciones sobre el mercado informal de arrendamiento residencial en Bogotá. Los resultados que se presentan a continuación fueron producto de la aplicación de una encuesta en el 2013 a arrendadores y arrendatarios que residían en el barrio Patio Bonito I, autor agradece los comentarios hechos en el proceso de evaluación del artículo.

* Doctor en Planeación Urbana y Regional del Instituto de Pesquisa e Planejamento Urbano Regional de la Universidad Federal de Río de Janeiro IPPUR-UFRJ, Brasil. Magister en Planeación y Administración del Desarrollo Regional del CIDER, Universidad de Los Andes, Colombia. Economista de la Universidad Nacional de Colombia. Entre los temas de investigación que trabaja se encuentran las dinámicas de segregación socioespacial, los mercados de suelo y vivienda y la movilidad residencial. ORCID: bttp://orcid.org/00000003-4166-317X

${ }^{1}$ La última actualización de la estratificación urbana en Bogotá fue adoptada mediante el Decreto 291 del 2013. Su principal utilidad radica en la posibilidad de establecer tarifas diferenciales en el cobro de los servicios públicos domiciliarios. Asi, mientras los hogares que viven en estratos uno, dos y tres son beneficiados con subsidios a las tarifas que pagan, los hogares que viven en estratos cinco y seis realizan una contribución adicional a su tarifa.

2 El aumento de la población que vive en arriendo $\Longrightarrow$

territarias 38 
también se ha visto en los sectores con ingresos medios $y$ altos: en estrato cuatro se pasó de 27,5\% a 33,1\%; en estrato cinco, de $18,5 \%$ a $28,7 \%$, y en estrato seis, de 20,9\% a 32,4\% (Secretaría Distrital de Planeación, 2015).

${ }^{3}$ Los estudios existentes en este tema pueden agruparse en tres grandes áreas: la caracterización del mercado, (Torres é Pérez, 2008; Blanco \& Volpe, 2015; Briceño-León, 2010), el análisis de los precios y lastransacciones (Parias, 2008), y la regulación a través de las politicaspúblicas (Blanco, Fretes Cibils es Muñoz Miranda, 2014; Gilbert, 1993).

4 La entidad encargada de regular las actividades de arrendamiento residencial en la ciudad es la Subsecretaría de Inspección, Vigilancia y Control de Vivienda. Esta entidad recopila información relacionada con las actividades de enajenación (venta) o arrendamiento de inmuebles destinados a vivienda, y para ello construye un Registro con información de las personas naturales 0 juridicas que se dedican a esta actividad económica, siendo uno de los criterios para dicha clasificación el haber establecido contratos de arrendamiento para un total de cinco o más

\section{territarias 38}

el cual está localizado al suroccidente de la ciudad.

$\mathrm{El}$ argumento central de esta investigación consiste en identificar en este tipo de mercado la existencia de vínculos sociales previos entre los arrendatarios y los arrendadores, y la generación de compromisos entre ellos para garantizar el funcionamiento de este tipo de mercado habitacional. Esta situación contrasta con la de un mercado en donde compiten agentes indiferenciados que deben recurrir a la generación de contratos para resolver la desconfianza que impera cuando las transacciones tienen lugar en condiciones de anonimato.

En la primera parte de este documento se presenta una breve descripción de la metodología empleada y luego se muestran los resultados de investigación centrados en la existencia de redes de relaciones y en la elaboración de compromisos entre los agentes económicos. Se cierra este documento con algunas conclusiones preliminares.

\section{Referentes teóricos y metodología}

Abramo (2002, 2009) plantea la existencia de tres lógicas de acción social por las cuales es posible entender los mecanismos de acceso al suelo y a la vivienda por parte de las familias de menores ingresos en las ciudades latinoamericanas. Se trata de las lógicas de Estado, Mercado y Necesidad. En lo que respecta a la lógica de mercado se distinguen a su vez dos subtipos que son el mercado formal y el mercado informal.
El criterio que se establece para esta diferenciación son los aspectos jurídicos que envuelven las transacciones que realizan los agentes económicos. Cuando se dan conforme a la normatividad oficial son denominados formales, y en caso contrario, informales. Si tenemos en cuenta que los hogares con bajos ingresos no acceden únicamente por la vía del mercado de compra y venta de lotes, o de viviendas ya construidas, sino que lo hacen también por la vía del arrendamiento, puede entonces diferenciarse un mercado formal y otro informal de arrendamiento residencial.

Bogotá es una ciudad en la cual existe una alta informalidad en cuanto al mercado de arrendamiento residencial que tiene lugar en barrios populares (Parias \& Abramo, 2009). Si bien un mercado de este tipo se puede dar independientemente del origen del barrio, se tiende a constatar que en aquellos de origen informal es frecuente la informalidad en el mercado de vivienda en arrendamiento ${ }^{4}$.

Con el fin de analizar la informalidad contractual en este tipo de mercado residencial, se realizó un análisis de caso tomando como criterios para la selección del barrio el proceso de constitución (origen informal) y la existencia de un mercado significativo de arrendamiento residencial. Partiendo de la existencia de estudios anteriores (Parias \& Abramo, 2009; Sáenz, 2009 ) se seleccionó el barrio Patio Bonito I, localizado al suroccidente de la ciudad. Según los datos del Censo del 2005, en este asentamiento vivían 10598 personas. La tabla 1 resume otros datos demográficos 
del barrio cuya extensión aproximada es de quince hectáreas en las cuales se disponen 43 manzanas y un poco más de mil lotes.

La tabla 1 nos permite comparar algunos indicadores socio-demográficos del barrio de estudio y de Bogotá. En términos muy generales se puede concluir que Patio Bonito I se caracteriza por tener una población joven, una presencia de población migrante y hogares con jefatura femenina, que son superiores a los datos observados en la ciudad. En cuanto a la tenencia de la vivienda, la proporción de hogares arrendatarios es superior frente al dato observado para Bogotá, y más aún la práctica de compartir vivienda, siendo el tipo predominante la casa.

El formulario de encuesta aplicado tuvo como objetivo analizar el funcionamiento del mercado de arrendamiento, para lo cual se dividió en cuatro partes: la primera examinó las características del jefe de hogar y de su familia; en segundo lugar, las características de la vivienda; en tercer lugar, las de la movilidad residencial; y en cuarto lugar, las de los contratos de arrendamiento. Este formulario se construyó a partir de los aportes de trabajos anteriores, como el de Parias \& Abramo (2009), Lacerda et al. (2010) y Lacerda (2011). En este documento se hace énfasis en la cuarta parte de dicho formulario.

La muestra de esta investigación se compone de 201 formularios aplicados en Patio Bonito I. La recolección de la información se realizó a partir del recorrido total del barrio, tratando de identificar en primer lugar posibles arrendadores, $y$ solo en caso de ser negativa la respuesta se indagaba por la presencia de hogares viviendo en arriendo. Del conjunto total de encuestas, $74(36,8 \%)$ corresponden a arrendadores y $127(63,2 \%)$ a arrendatarios, lo cual presenta cierta semejanza con los datos censales presentados en la tabla $1^{5}$.

\section{Las redes de relaciones y su importancia para el mercado de arrendamiento en Patio Bonito}

La presencia de informalidad contractual suele asociarse como causa de la inseguridad en la tenencia de la vivienda. El argumento que sustenta esta afirmación es que dado que no existe una clara definición de derechos de propiedad, los arrendatarios se encontrarían expuestos a ser desalojados en cualquier momento o, en el caso de los arrendadores, a tener dificultades para recuperar el espacio arrendado. Se trata de problemas que en la literatura económica se conocen como costos de transacción (Bejarano, 1999; Coase, 1960/1994; Younkis, 2000; Fiani, 2002/2011).

Diferentes autores han tratado de analizar la forma como opera el sistema económico. Para esta investigación se tomará como referente la Escuela Francesa de las Convenciones, que propone un abordaje institucional heterodoxo de los mercados (Favereau, 1993) ${ }^{6}$. La premisa más importante plantea que el mercado se encuentra incrustado en la sociedad, lo que lleva a situar las relaciones económicas en el contexto social, ya que estas precisan unidades habitacionales (Secretaria de Hábitat, 2014). Este criterio excluye por tanto aquellas actividades de arrendamiento de menor magnitud, así como a quienes no siguen las normativas oficiales. Una de las consecuencias de este subregistro es la ausencia de información sobre el total de contratos que se establecen en este mercado y sobre la proporción que se podría denominar informal.

${ }^{5}$ Inicialmente se habia determinado una muestra de 215 encuestados y se habia realizado un proceso de selección de manzanas y de lotes para la aplicación de la encuesta. Sin embargo, la alta tasa de rechazo experimentada al comienzo de la aplicación llevó a modificar rápidamente ese criterio y a optar por tomar el conjunto total de manzanas y viviendas. Al final, la muestra se encuentra distribuida de manera uniforme en el conjunto total de manzanas del barrio, con lo cual se minimizan los sesgos en los resultados de la encuesta.

6 Existen otras propuestas interpretativas como la de estructuras de gobernanza (Williamsom, 1986/1996) y la de convenciones y nor mas sociales en su vertiente anglosajona (Bowles, 2004; North, 1999). La selección de la propuesta

territarias 38 
de convenciones de la Escuela Francesa obedece a su postura critica frente a las visiones más ortodoxas del análisis económico, que no cuestionan la racionalidad de los agentes ni reconocen el incrustamiento del mercado en lo social; por el contrario, terminan siendo visiones economicistas que incorporan categorias de análisis sociológico sin modificar la premisa de agentes que actúan bajo racionalidad paramétrica.

\section{territarias 38}

Tabla 1. Principales indicadores censales: Patio Bonito I (Censo 2005)

\begin{tabular}{|c|c|c|c|c|}
\hline \multirow[b]{2}{*}{ Personas } & \multicolumn{2}{|c|}{ Patio Bonito I } & \multicolumn{2}{|c|}{ Bogotá } \\
\hline & & $\%$ & & $\%$ \\
\hline Menores de 15 años & 3037 & 28,7 & 1793039 & 26,5 \\
\hline Entre 20 y 34 años & 3008 & 28,4 & 1797344 & 26,6 \\
\hline Mayores de 60 años & 720 & 6,8 & 562896 & 8,3 \\
\hline Nacidos en Bogotá & 5308 & 50,1 & 4020717 & 59,4 \\
\hline Nacidos fuera de Bogotá & 5282 & 49,8 & 2502612 & 37,0 \\
\hline Hace cinco años vivía en Bogotá & 9886 & 93,3 & 5612432 & 83,0 \\
\hline Hace cinco años vivía en otra ciudad & 707 & 6,7 & 357548 & 5,3 \\
\hline Total & 10598 & & 6763325 & \\
\hline \multicolumn{5}{|c|}{ Familias } \\
\hline Tipo de familia & & $\%$ & & $\%$ \\
\hline Unipersonal & 368 & 12,3 & 256984 & 13,3 \\
\hline Nuclear & 1721 & 57,4 & 1150131 & 59,7 \\
\hline Compuesta & 262 & 8,7 & 110490 & 5,7 \\
\hline Extensa & 645 & 21,5 & 409785 & 21,3 \\
\hline Total & 2996 & 100,0 & 1927390 & \\
\hline Familias arrendatarias* & 2092 & 63,5 & 836857 & 43,4 \\
\hline Familias propietarias ${ }^{*}$ & 1202 & 36,5 & 895700 & 46,5 \\
\hline \multicolumn{5}{|l|}{ Otros } \\
\hline Familias con seis personas o más & 280 & 9,3 & 190961 & 9,9 \\
\hline Familias que comparten vivienda & 1388 & 46,3 & 154831 & 8,0 \\
\hline Familias con jefatura femenina & 1122 & 37,4 & 617439 & 32,0 \\
\hline \multicolumn{5}{|l|}{ Tipo de vivienda } \\
\hline Casa & 1158 & 53,7 & 704695 & 40,1 \\
\hline Apartamento & 698 & 32,3 & 892455 & 50,8 \\
\hline Cuarto & 302 & 14,0 & 161194 & 9,2 \\
\hline Total & 2158 & & 1758344 & \\
\hline
\end{tabular}

Fuente: Censo 2005, microdatos. Los datos para Bogotá fueron producidos por Salas, Dureau \& Le Roux. Los datos para Patio Bonito I fueron procesados por el autor.

"Los datos relacionados con tenencia de la vivienda corresponden a un sector censal que agrupa, además de Patio Bonito I, a otros barrios del sector, pero se considera una buena aproximación. 
de la confianza entre las personas y de una estructura social que las apoye.

Desde esta propuesta, los agentes económicos son vistos como individuos capaces de realizar acuerdos y de establecer relaciones de cooperación que los llevan a comprometerse en escenarios complejos e imprevisibles. Existen dos niveles de coordinación: las reglas y las acciones individuales. Las convenciones son entonces formas colectivas que aseguran la compatibilidad entre las acciones individuales; y a su vez existe una diversidad de acuerdos convencionales: el precio es apenas uno de ellos (Eymard-Duvernay, 2001).

A partir de este referencial teórico vale la pena considerar si las relaciones entre arrendatarios y arrendadores pueden abordarse desde el concepto de compromiso, que puede entonces constituirse en una convención, y de esa forma garantizar el funcionamiento de este tipo de mercado residencial. Para explorar esta hipótesis se requiere, sin embargo, indagar por la existencia de aspectos como las redes de relaciones (Granovetter, 1985) entre los agentes económicos y su importancia para el surgimiento de mecanismos de cooperación basados en una expectativa de reciprocidad.

En el caso del barrio analizado se indagó por la existencia de redes de relaciones entre arrendatarios y propietarios-arrendadores. La tabla 2 presenta información sobre el lugar de nacimiento de los encuestados. Los resultados muestran el predominio de arrendatarios y arrendadores que comparten el mismo lugar de nacimiento, imperando de un lado aquellos que nacen en la región andina más próxima a Bogotá, y del otro los nacidos en la misma ciudad. En lo que atañe al primer grupo se destaca la participación de una población nacida en los departamentos de Cundinamarca y Boyacá7.

El paisanaje es un aspecto importante porque sirve para entender que una fuente de conflicto observada entre los agentes surge a raíz de pautas culturales regionales que son diferentes en relación con el uso de la vivienda. En Patio Bonito I, la presencia de una migración que proviene de zonas como el Caribe alcanza un 9,5\% entre la población arrendataria. $\mathrm{Al}$ respecto es importante señalar que dicha población tiene mayores dificultades para acceder a vivienda en arriendo, ya que predominan conflictos ocasionados por comportamientos que no se ajustan a las pautas esperadas por los arrendadores ${ }^{8}$.

La existencia de redes de relaciones requiere de tiempo para su consolidación; es por ello que además del rol que puede tener el hecho de compartir el mismo sitio de origen se necesite de tiempos prolongados de permanencia en el barrio para la generación de vínculos sociales no solo entre propietarios-arrendadores sino entre estos y los arrendatarios. Para examinar esta relación se puede indagar por el tiempo de permanencia en el barrio y en la ciudad de estos agentes económicos. Se tomarán, a manera de ejemplo, aquellos arrendadores y arrendatarios que nacieron en Bogotá,
Del total de arrendadores un $43,3 \%$ se concentraba en estos dos departamentos, mientras que entre los arrendatarios la proporción alcanzaba un 26,8\%.

${ }^{8}$ Durante la aplicación de las encuestas algunos arrendadores expresaron que un criterio para elegir a un arrendatario era el lugar de procedencia. Con esto se referian explicitamente a no quererle arrendar a la población costeña, ya que muchas veces era incumplida en el pago y, si bien se le arrendaba para un numero especifico de personas, en ella persistía la práctica de acoger a otros miembros de su familia, con lo cual se incrementaba el consumo de agua y demás servicios públicos. En los recorridos realizados en el barrio se observó que tendia a existir cierta espacialización de esta población en determinadas calles, lo que daba indicios de una división social del espacio en una escala más micro.

territarias 38

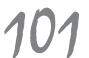


${ }^{9}$ Por motivos de extensión no se presentan los resultados para los arrendadores $y$ arrendatarios que nacieron en otras regiones del pais que están más alejadas de Bogotá. Se ha realizado el ejercicio teniendo en cuenta que la mayoría de encuestados pertenece a estas regiones. En el caso de los arrendadores, el $90 \%$ corresponde a estos dos lugares de nacimiento, mientras que entre los arrendatarios se trata del 73,3\%.

10 Patio Bonito I es el resultado de un proceso de comercialización de lotes sin urbanizar a comienzos de los años setenta. A través de la Junta de Acción Comunal se desarrollaron diferentes actividades para satisfacer las necesidades de la población. En 1979 el barrio padeció los estragos de una inundación, pero lejos de la reubicación de las familias se dio un proceso de consolidación en los años posteriores, que no solo fue producto de la legalización del barrio y de la intervención de programas de urbanización por parte del Estado, sino de las mismas actividades de sus habitantes, quienes ampliaron sus viviendas al construir pisos adicionales y del surgimiento de una centralidad económica de tipo popular en las principales vías del barrio (Diaz, 2003).

territarios 38

Tabla 2. Distribución según la región de origen de los arrendadores y arrendatarios encuestados

\begin{tabular}{|l|c|c|}
\hline \multicolumn{1}{|c|}{ Región de Colombia } & Arrendadores & Arrendatarios \\
\hline Bogotá & 33,8 & 33,1 \\
\hline $\begin{array}{l}\text { Región andina de la cordillera oriental (Cundinamarca, Boyacá, Santander, } \\
\text { Norte de Santander, Tolima, Meta) }\end{array}$ & 56,8 & 40,2 \\
\hline $\begin{array}{l}\text { Región andina de las cordilleras central y occidental (Antioquia, Caldas, } \\
\text { Risaralda, Quindío, Valle del Cauca) }\end{array}$ & 3,9 & 15,0 \\
\hline Región sur (Huila, Cauca, Nariño) & 4,0 & 2,4 \\
\hline Región norte (caribe) (Cesar, Bolívar, Córdoba, Magdalena) & 1,3 & 9,5 \\
\hline Total & 100,0 & 100,0 \\
\hline
\end{tabular}

Fuente: Elaboración propia con base en datos de los cuestionarios realizados en Patio Bonito I, septiembre del 2013.

así como quienes nacieron en los departamentos más próximos a la capital (categoría cordillera oriental de la tabla 2$)^{9}$.

Los arrendadores que nacieron en los departamentos más cercanos a Bogotá (gráfico 1) conforman una población que lleva más de veinte años viviendo en esta ciudad, y en Patio Bonito I, la cual corresponde a la población originaria del barrio $^{10}$. En el caso de los arrendatarios que nacieron en estos mismos departamentos (gráfico 2), se observa una mayor diversidad en cuanto a tiempo de residencia en la ciudad, y especialmente en Patio Bonito I. La proporción de arrendatarios que llevan más de veinte años disminuye, pero sigue siendo la más importante. Además se señala que entre esta población se presenta una mínima movilidad en términos residenciales. Por último, con referencia a quienes nacieron en Bogotá (gráfico 3 ) se observa un patrón similar. Mientras entre los propietarios arrendadores hay una alta proporción de quienes llevan más de veinte años en el barrio, cuando se examina el caso de los arrendatarios la proporción cae a la mitad.

Un aspecto importante para destacar de los gráficos corresponde a la población arrendataria que lleva menos de cinco años y que tiene un peso muy cercano al de los arrendatarios que llevan más de veinte años en el barrio. La llegada de estos arrendatarios en los últimos años a Patio Bonito I muestra que la oferta de espacio en alquiler permanece activa. Como un barrio de origen informal donde la construcción de la vivienda es progresiva, la generación de nuevos espacios para alquilar puede tener lugar aún. Dicha oferta puede surgir no 
solo por la densificación de las viviendas sino también por su crecimiento vertical (construcción de nuevos pisos).

Los gráficos 1, 2 y 3 nos permiten entonces plantear que los arrendadores y arrendatarios encuestados en Patio Bo- nito I comparten entre sí aspectos como el mismo lugar de nacimiento y periodos prolongados de residencia en el mismo barrio. Sin embargo, se observan algunas diferencias que si bien no son muy significativas en términos porcentuales indican

Gráfico 1. Tiempo de residencia en Bogotá y en Patio Bonito I de la población arrendadora que nació en departamentos de la cordillera oriental



— Permanencia en Patio Bonito I

- Permanencia en Bogotá

Fuente: Elaboración propia con base en datos de los cuestionarios practicados en Patio Bonito I, septiembre del 2013. El número de observaciones para la permanencia en Patio Bonito I es de 42, y para la permanencia en Bogotá es de 41.

Gráfico 2. Tiempo de residencia en Bogotá y en Patio Bonito I de la población arrendataria que nació en departamentos de la cordillera oriental



Fuente: Elaboración propia con base en datos de los cuestionarios practicados en Patio Bonito I, septiembre del 2013. El número de observaciones para la permanencia en Patio Bonito I es de 51, mientras que para la permanencia en Bogotá es de 48. 
una heterogeneidad, especialmente entre la población de arrendatarios. Esto debido a que en las últimas décadas llegaron personas de regiones más alejadas del país, como la región caribe, el sur del país, y la región central (tradicionalmente llamada región cafetera).

Un último aspecto adicional que se indagó en la encuesta se refiere a la presencia de lazos de parentesco territorializados en el barrio. La tabla 3 permite observar la existencia e importancia de permanecer cerca de los familiares para la población encuestada en Patio Bonito I.

En el caso de la población encuestada la cercanía a los familiares es el primer motivo para permanecer en el barrio, mucho más que la cercanía a amigos. Además, el peso de las redes en la elección residencial supera a factores como la proximidad al lugar de trabajo, que se destaca en los aná- lisis más convencionales sobre movilidad residencial. Como ya se ha observado en otras investigaciones, la proximidad a las redes de relaciones y su territorialización en el barrio (estrategias residenciales territorializadas) (Parias \& Abramo, 2009) constituyen una de las principales características en la conformación de la identidad de los sectores populares y, por ende, en la definición de las relaciones contractuales que tienen lugar en el mercado de arrendamiento residencial.

La tabla 4 confirma esta situación de proximidad, ya que la localización de los familiares y del amigo más frecuentado por parte de los encuestados corresponde al mismo barrio o a barrios muy próximos a Patio Bonito I. Un ejemplo de ello es, por ejemplo, que en el caso de los arrendadores y arrendatarios el amigo más frecuentado vive en el mismo barrio. En el caso de los

\section{territarias 38}

Gráfico 3. Tiempo de residencia en Patio Bonito I de la población arrendataria y arrendadora que nació en Bogotá



Fuente: Elaboración propia con base en datos de los cuestionarios aplicados en Patio Bonito I, septiembre del 2013. El número de observaciones en arrendatarios es de 42 , mientras que en arrendadores es de 16. 
Tabla 3. Razones por las cuales permanece en el barrio (arrendadores y arrendatarios)

\begin{tabular}{|l|c|c|}
\hline \multicolumn{1}{|c|}{ Razones para permanecer en el barrio } & $\begin{array}{c}\text { Porcentaje } \\
\text { arrendadores* }\end{array}$ & $\begin{array}{c}\text { Porcentaje } \\
\text { arrendatarios }\end{array}$ \\
\hline Por cercanía a los amigos & 17,6 & 11,8 \\
\hline Por cercanía a parientes & 35,1 & 43,3 \\
\hline Por la proximidad al lugar de trabajo & 14,9 & 21,3 \\
\hline Por la oportunidad de ser propietario (aplica para arrendadores) & 56,8 & 17,3 \\
\hline Por los arriendos baratos (aplica para arrendatarios) & & \\
\hline Por la realización de algún trabajo comunitario & 5,4 & \\
\hline Porque es una opción de inversión & 6,8 & 1,6 \\
\hline Para arrendar o tener un negocio en la vivienda & 14,9 & 9,5 \\
\hline Por desplazamiento forzado por la guerra (aplica para arrendatarios) & & 6,3 \\
\hline Por otros motivos & 8,1 & 1,4 \\
\hline NS/NR & & \\
\hline
\end{tabular}

Fuente: Elaboración propia con base en los datos de los cuestionarios realizados en Patio Bonito, septiembre del 2013.

* La suma de los porcentajes no es igual a cien porque una persona puede indicar más de una razón.

familiares, el grado de proximidad es más difuso; por ejemplo, entre los arrendadores se da con los padres de los encuestados, y entre los arrendatarios, con sus hermanos.

La existencia de las redes de relaciones basadas en el parentesco también explica la localización residencial, si se toma en cuenta el lugar donde residen los familiares más próximos (padres, hijos, hermanos), lo cual apoya también la hipótesis de que son estas redes las más importantes para que circule la información acerca de los espacios ofertados. Una particularidad en el caso de los arrendadores es la presencia de redes apoyadas en la vecindad, ya que se destaca en esta población la existencia de amigos que se visitan en el mismo barrio.

Estas redes, sin embargo, no implican una mayor participación en asuntos comunitarios, pues del total de encuestados solo el $33,8 \%$ de los arrendadores y el $28,2 \%$ de los arrendatarios afirmó participar de alguna organización, principalmente religiosa (lo cual se restringe incluso al hecho de asistir a misa o a culto los domingos). Lo más paradójico en términos de relaciones de vecindario aparece cuando se indaga por la relación que se tiene con los vecinos: al respecto, las percepciones son positivas por el hecho de que no se tiene 
Tabla 4. Lugar donde viven los familiares y el amigo que más frecuentan

\begin{tabular}{|c|c|c|c|c|c|c|c|c|}
\hline Lugar & \multicolumn{2}{|c|}{ En el mismo barrio } & \multicolumn{2}{|c|}{$\begin{array}{l}\text { En otro barrio de la } \\
\text { alcaldía local }\end{array}$} & \multicolumn{2}{|c|}{ En otra localidad } & \multicolumn{2}{|c|}{$\begin{array}{c}\text { Número de } \\
\text { observaciones }\end{array}$} \\
\hline \multicolumn{9}{|c|}{ Arrendadores } \\
\hline & Frecuencia & $\%$ & Frecuencia & $\%$ & Frecuencia & $\%$ & Total & $\%$ \\
\hline Padres & 7 & 70,0 & 1 & 10,0 & 2 & 20,0 & 10 & 100,0 \\
\hline Hermanos & 7 & 15,6 & 15 & 33,3 & 23 & 51,1 & 45 & 100,0 \\
\hline Tíos & 2 & 33,3 & 0 & 0,0 & 4 & 66,7 & 6 & 100,0 \\
\hline Hijos o nietos & 2 & 22,2 & 5 & 55,6 & 2 & 22,2 & 9 & 100,0 \\
\hline Otro familiar & 2 & 18,2 & 1 & 9,1 & 8 & 72,7 & 11 & 100,0 \\
\hline Amigo & 27 & 60,0 & 9 & 20,0 & 9 & 20,0 & 45 & 100,0 \\
\hline \multicolumn{9}{|c|}{ Arrendadores } \\
\hline & Frecuencia & $\%$ & Frecuencia & $\%$ & Frecuencia & $\%$ & Total & $\%$ \\
\hline Padres & 10 & 37,0 & 6 & 22,2 & 11 & 40,7 & 28 & 100,0 \\
\hline Hermanos & 30 & 41,7 & 18 & 25,0 & 24 & 33,3 & 72 & 100,0 \\
\hline Tíos & 6 & 20,0 & 8 & 26,7 & 16 & 53,3 & 30 & 100,0 \\
\hline Hijos o nietos & 2 & 66,7 & 0 & 0,0 & 1 & 33,3 & 3 & 100,0 \\
\hline Otro familiar & 6 & 23,1 & 6 & 23,1 & 14 & 53,8 & 26 & 100,0 \\
\hline Amigo & 48 & 61,5 & 16 & 20,5 & 14 & 18,0 & 78 & 100,0 \\
\hline
\end{tabular}

Fuente: Elaboración propia a partir de los datos de los cuestionarios realizados en Patio Bonito I.

* El total puede ser menor que el número total de la muestra en virtud de que no todos realizan la actividad señalada.

mayor contacto entre sí - en palabras de una entrevistada - porque "nadie se mete con nadie".

La utilidad de las redes de relaciones a la luz de comentarios como el de la encuestada parece poner en duda hasta dónde se despliega en el barrio una sociabilidad primaria que fomente la participación ciudadana en los asuntos que van más allá del espacio propio del hogar (la vivienda), y por lo tanto en la generación de una identidad y movilización por el territorio, sobre todo entre los arrendatarios. Hataya (2009) señala que en virtud del tipo de tenencia es posible comprender la poca o nula participación comunitaria de los arrendatarios, ya que dicha población se encontraría de forma transitoria en el 
barrio; pero como se observa para Patio Bonito I, existe una población arrendataria que ha vivido por décadas allí. Se requeriría explorar otras hipótesis para explicar el porqué de esta baja participación, diferente a la asociación entre el tipo de tenencia y el tiempo de residencia en el barrio.

En este escenario se tiene entonces que hay varias similitudes entre los arrendatarios y los propietarios-arrendadores; sin embargo, se requiere analizar con mayor detalle el tipo de relación contractual que establecen los participantes en este mercado, si se quiere identificar la existencia de redes de relaciones y su importancia para la generación de formas de cooperación basadas en el compromiso.

\section{Convenciones y generación de compromisos entre los agentes económicos}

Una posibilidad de análisis de las relaciones contractuales en los mercados de arrendamiento residencial surge a partir de los planteamientos de la Escuela Francesa de las Convenciones, que plantea el concepto de compromiso para dar cuenta del proceso por el cual se establecen las convenciones.

La constitución de un compromiso en convención requiere de cinco fases o etapas: en primer lugar, la llamada fase de compromisos mutuos, en donde los individuos se comprometen a cooperar alrededor de un objetivo específico. Como el ambiente es demasiado imprevisible, no se dan las condiciones para que se pueda establecer un contrato perfecto. Una vez asumido el compromiso, tiene lugar una fase de interacción a la vista ya que las personas están muy próximas. En esa situación hay un grado relativo de inexactitud y es posible, por lo tanto, realizar los ajustes necesarios en función de dicha proximidad. La tercera fase es aquella donde opera propiamente la convención, ya que se hacen explícitos los compromisos (se establece un acuerdo separado de la acción). La explicitación no necesariamente es escrita, pues puede ser un intercambio verbal, y es a través del lenguaje que es posible también expresar la insatisfacción y el inconformismo, que puede ser superado a través del diálogo ${ }^{11}$ (Eymard-Duvernay, 2001). La cuarta fase es la actualización, que se refiere a la realización de ajustes locales fruto de la interpretación de las reglas que generan arreglos, las cuales se estiran un poco. Estos ajustes pueden salirse de la regla pero sirven para restablecer los acuerdos. Por último se encuentra la fase de revisión de las convenciones, que se trata del momento en que tiene lugar el aprendizaje organizacional, que se caracteriza por el cambio de las reglas en virtud de los problemas surgidos y de las formas empleadas para su resolución (Eymard-Duvernay, 2001).

Tomando este referente es posible comprender algunas características de las relaciones contractuales entre arrendatarios y arrendadores en Patio Bonito I. En primer lugar, el gráfico 4 presenta información sobre los mecanismos a través de los cuales circula la información sobre oferta de lugares para tomar en arrendamiento. Un $56,7 \%$ de los arrendatarios conoció de la
${ }^{11}$ Ese reconocimiento de la explicitación a través del lenguaje ya habia sido identificado y analizado por Albert Hirschmann -quién publicó en 1977 una obra llamada Salida, voz y lealtad-, el cual es recuperado por la Escuela Francesa de las Convenciones. territarios 38 107 
oferta gracias a un amigo o familiar, mientras que entre los arrendadores se acudió a este mecanismo para difundir su oferta en un $52,8 \%$. Este resultado significa que un papel de las redes de relaciones territorializadas en el barrio está asociado con esta circulación de la información.

La importancia de las redes de relaciones se constata también cuando se indaga entre la población encuestada sobre la existencia de una relación previa entre arrendatarios y arrendadores. Un $48 \%$ de los arrendatarios que se encuestaron respondió afirmativamente a esta pregunta. Es importante, sin embargo, tener presente que, del otro lado, un $52 \%$ afirmó que no presentaba relación alguna, y en ese sentido existe cierto grado de anonimato o desconocimiento entre arrendadores y arrendatarios.

Las modalidades más anónimas surgen cuando el arrendador coloca el aviso en la ventana y atiende personalmente a los candidatos que se acercan con la intención de conocer las características del inmueble ofertado. Durante la aplicación de los cuestionarios se observaron personas (generalmente mujeres) que caminaban por las calles del barrio buscando vivienda. En ese primer encuentro tiene lugar una entrevista que es muy importante para establecer un interés entre las partes, ya que mientras que el arrendador presenta los espacios de la vivienda en oferta, indaga sobre las características del hogar: número de personas, actividad profesional, lugar de procedencia, razones por las cuales está buscando, etc. Muchos dicen que en ese primer contacto ya es posible saber si se trata de gente de confiar o no. La existencia de una alta demanda por espacio para tomar en arriendo les permite también a los arrendadores aplazar la elección de un candidato que reúna las condiciones ade-

Gráfico 4. Modalidad por la cual la familia conoció de la existencia de la oferta

\section{territarias 38} en arriendo del sitio en el que reside en Patio Bonito I

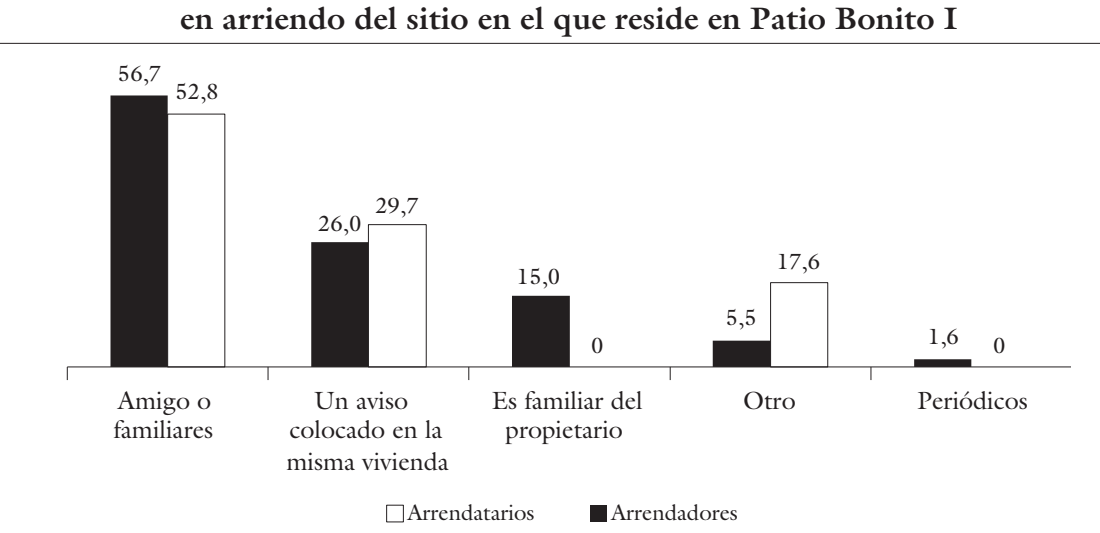

Fuente: Elaboración propia con base en los datos de los cuestionarios realizados en Patio Bonito I, septiembre del 2013 * Debido a que una persona puede emplear más de un mecanismo, la suma de los porcentajes no es igual a cien. 
cuadas. Dicha situación se ve reforzada cuando se viven experiencias negativas con anteriores inquilinos, que llevan entonces a abandonar los mecanismos más indirectos e inmediatos de divulgación de la oferta y a recurrir a las redes de relaciones de las que hacen parte, con la expectativa de encontrar un inquilino que acate las normas establecidas por el arrendador.

El gráfico 5 y la tabla 5 resumen lo que podría considerarse como el grado de personalización de la relación contractual y el tipo de contrato que predomina en Patio Bonito I. Una relación personalizada implica en este caso la existencia de una relación previa entre arrendador y arrendatario. La existencia de esa personalización es muy importante, sobre todo cuando los contratos son de palabra, y en ese sentido tiende a predominar más este tipo de contratos frente a aquellos que son escritos y registrados en notaria. Un bajo grado de personalización se asocia con la ausencia de relaciones previas a la celebración del contrato. Cuando tiene lugar este anonimato se tiende más a apelar a los contratos escritos y registrados en notaria.

Es importante señalar que la existencia de compromiso entre las partes no significa ausencia de contratos. La ley colombiana reconoce la existencia de contratos, de palabra y escritos, y la posibilidad de acudir al Estado en caso de incumplimiento por alguna de las partes. Lo que se quiere

Tabla 5. Tipo de contrato según el grado de personalización de la relación entre arrendatarios y arrendadores

\begin{tabular}{|c|c|c|c|c|c|c|c|}
\hline & \multicolumn{2}{|c|}{ Contrato oral } & \multicolumn{2}{|c|}{ Contrato escrito } & \multicolumn{2}{|c|}{$\begin{array}{l}\text { Contrato escrito y } \\
\text { registrado en notaría }\end{array}$} & Total \\
\hline \multicolumn{8}{|c|}{ Arrendatarios } \\
\hline Relación con el arrendador & Frecuencia & $\%$ & Frecuencia & $\%$ & Frecuencia & $\%$ & $\%$ \\
\hline Ya se conocían & 43 & 71,7 & 14 & 23,3 & 3 & 5 & 100,0 \\
\hline No se conocían & 32 & 50 & 26 & 40,6 & 6 & 9,4 & 100,0 \\
\hline \multirow[t]{2}{*}{ Total } & 75 & 60,5 & 40 & 32,3 & 9 & 7,3 & 100,0 \\
\hline & \multicolumn{2}{|c|}{ Contrato oral } & \multicolumn{2}{|c|}{ Contrato escrito } & \multicolumn{2}{|c|}{$\begin{array}{l}\text { Contrato escrito y } \\
\text { registrado en notaría }\end{array}$} & Total \\
\hline \multicolumn{7}{|c|}{ Arrendadores } & \\
\hline $\begin{array}{l}\text { Tipo de oferta utilizada por } \\
\text { el arrendador }\end{array}$ & Frecuencia & $\%$ & Frecuencia & $\%$ & Frecuencia & $\%$ & $\%$ \\
\hline Oferta de aviso en la ventana & 14 & 50 & 12 & 42,9 & 2 & 7,1 & 100,0 \\
\hline Oferta personalizada & 27 & 58,7 & 18 & 39,1 & 1 & 2,2 & 100,0 \\
\hline
\end{tabular}

Fuente: Elaboración propia a partir de los datos de los cuestionarios realizados en Patio Bonito I. 


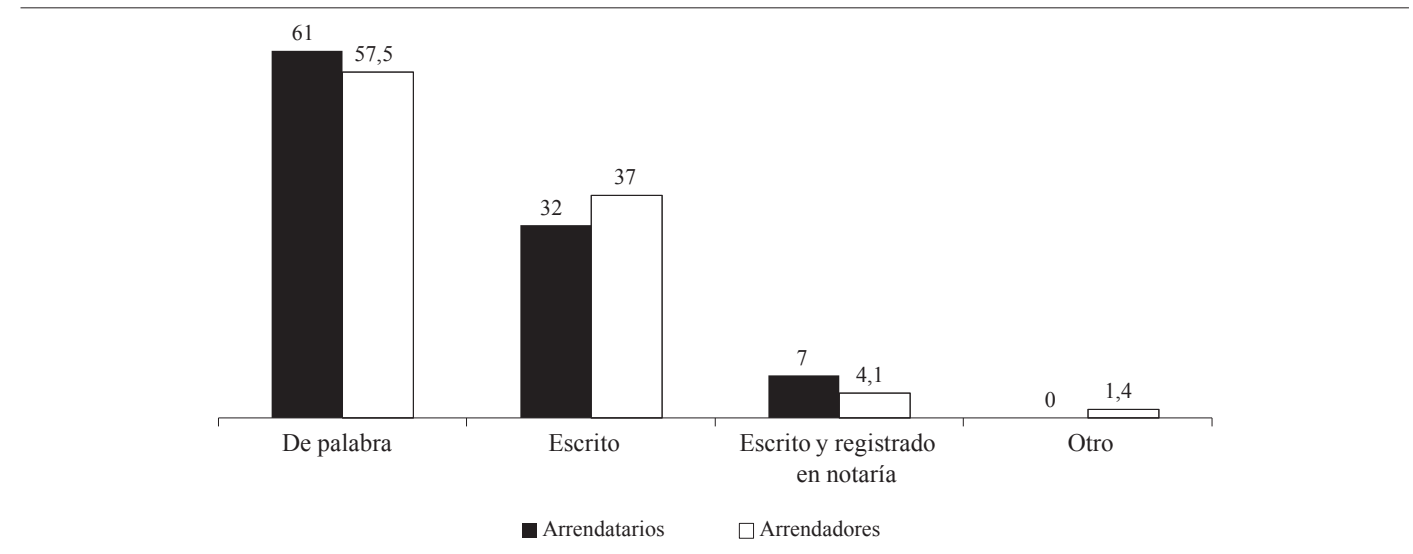

Fuente: Elaboración propia con base en los datos recopilados en los cuestionarios aplicados a la población de Patio Bonito I, septiembre del 2013.

mostrar con los datos relacionados con el grado de personalización es que las relaciones contractuales pueden ser objeto de una subjetivación (Sáenz, 2016). En ese sentido se abre la posibilidad para que otro tipo de ordenamientos jurídicos pueda entrar a operar, ya que la generación del compromiso como convención permite que las situaciones de inconformismo se denuncien y se resuelvan a través del diálogo entre las partes, quienes pueden redefinir los compromisos y actualizar dicha convención.

Entre los arrendadores que se encuestaron también se observa la tendencia a subjetivar la relación con el arrendatario. Cuando la oferta es personalizada predominan los acuerdos verbales, mientras que la proporción es menor en el caso de acuerdos escritos. En cuanto a las formas más próximas a un contrato (escrito y re- gistrado en notaria), estas son mayores en situaciones donde no existe esa personalización de la relación con el arrendatario y se parte de un grado elevado de anonimato.

Los arreglos jurídicos presentes en barrios como Patio Bonito I expresan la producción de un orden jurídico desde las comunidades y de su interrelación con el que es producido por el Estado. Dicho formalismo popular, que ha sido constatado en otras investigaciones (Magalhães, 2013), puede analizarse aquí al tomar en cuenta la existencia de esos compromisos que establecen los agentes económicos. Como se apreció en la investigación realizada, es posible adquirir un formato de contrato de arrendamiento en las papelerías del barrio y utilizarlo para dejar por escrito el acuerdo entre las partes. Dicho formato trae una descripción de la ley que regula dicho mercado (Ley 820 del 2003); 
sin embargo, para las partes es suficiente con la firma del documento y no exigen el registro en la notaria.

La utilización de esta forma escrita, sin embargo, tiende a ser abandonada en algunas ocasiones: cuando la confianza es alcanzada entre las partes y la relación económica se consolida con el tiempo. Es decir, el contrato escrito actúa de forma coyuntural como mecanismo para enfrentar posibles situaciones de oportunismo, pero una vez superado un periodo de prueba, se considera innecesario. La ausencia de un contrato escrito también es importante porque permite materializar, cuando sea necesario, la opción de salida y terminación unilateral del compromiso sin asumir los costos a los que se deberían enfrentar cuando se está dentro de la normativa oficial.

La tabla 6 nos permite observar la relación que existe entre las modalidades de contrato y el periodo de residencia que lleva el arrendatario en la vivienda, esto según el grado de personalización de la relación entre los agentes. Cuando existe una relación previa entre arrendatario y arrendador es predominante la modalidad oral, independientemente del tiempo que se lleve residiendo en la vivienda. En el caso de que la relación parte de un grado relativo de anonimato la proporción de contratos de palabra se reduce considerablemente y predominan los contratos escritos, aunque no necesariamente registrados en notaria. Esta distinción es importante porque se puede plantear entonces que lo que se denomina tradicionalmente como informalidad contractual en realidad im- plica una personalización o subjetivación de una relación como la mercantil, que tradicionalmente se entiende anónima. Hacer de una relación económica algo subjetivo significará la posibilidad de crear sistemas propios de regulación de este tipo de mercado, sin que se requiera necesariamente de la intervención del Estado.

La tabla 7 presenta la información desde el lado de los arrendadores encuestados. De los datos obtenidos se han considerado tres situaciones: la primera es considerada como la de mayor personalización de la relación contractual — en donde se arrienda a familiares y/o amigos - y llega a representar un $42 \%$ de los casos.

Una segunda situación corresponde a una personalización relativa o intermedia en la que se afirma arrendar a personas desconocidas pero recomendadas $\mathrm{y} / \mathrm{o}$ amigos tanto como a personas desconocidas. Esta es la que mayor peso tiene: alcanza el $53 \%$ de los casos. Por último figura una modalidad donde predomina el mayor grado de anonimato que representa tan solo un $5,4 \%$ del total de los casos, lo que muestra que no es tan fácil acceder a la vivienda sin pertenecer antes a alguna de red territorializada en el barrio o tener un conocido que permita la entrada a la red.

En síntesis: la información de las tablas 6 y 7 permiten comprender que la existencia de una relación personalizada, que antecede a la relación estrictamente económica entre arrendatarios y arrendadores, es importante en este mercado habitacional. El papel de las redes de relaciones es fundamental para que este mercado territarias 38

111 
Tabla 6. Tipo de contrato predominante según el tiempo de residencia del arrendatario en el domicilio y según el tipo de relación que posee con su arrendador

\begin{tabular}{|c|c|c|c|c|c|c|c|c|}
\hline \multirow{3}{*}{\begin{tabular}{|c|} 
Tipo de contrato \\
$\begin{array}{c}\text { Tiempo de residencia en } \\
\text { el domicilio actual }\end{array}$
\end{tabular}} & \multicolumn{8}{|c|}{ El arrendatario ya conocía a su arrendador (relación personalizada) } \\
\hline & \multicolumn{2}{|c|}{ Contrato oral } & \multicolumn{2}{|c|}{ Contrato escrito } & \multicolumn{2}{|c|}{$\begin{array}{l}\text { Contrato escrito y } \\
\text { registrado }\end{array}$} & \multicolumn{2}{|l|}{ Total } \\
\hline & Frecuencia & $\%$ & Frecuencia & $\%$ & Frecuencia & $\%$ & Frecuencia & $\%$ \\
\hline Menos de 1 año & 7 & 63,6 & 4 & 36,4 & 0 & 0 & 11 & 100 \\
\hline Entre 1 y 5 años & 20 & 64,5 & 8 & 25,8 & 3 & 9,7 & 31 & 100 \\
\hline Entre 5 y 10 años & 10 & 90,9 & 1 & 9,1 & 0 & 0 & 1 & 100 \\
\hline Más de 10 años & 6 & 85,7 & 1 & 14,3 & 0 & 0 & 7 & 100 \\
\hline \multirow[t]{2}{*}{ Total } & 3 & 71,7 & 14 & 23,3 & 3 & 5 & 60 & 100 \\
\hline & \multicolumn{8}{|c|}{$\begin{array}{l}\text { El arrendatario no se conocía con el arrendador } \\
\text { (relación despersonalizada o anónima) }\end{array}$} \\
\hline $\begin{array}{l}\text { Tiempo de residencia en } \\
\text { el domicilio actual }\end{array}$ & Frecuencia & $\%$ & Frecuencia & $\%$ & Frecuencia & $\%$ & Frecuencia & $\%$ \\
\hline Menos de 1 año & 11 & 50 & 10 & 45,5 & 1 & 4,5 & 22 & 100 \\
\hline Entre 1 y 5 años & 11 & 44 & 11 & 44 & 3 & 12 & 25 & 100 \\
\hline Entre 5 y 10 años & 9 & 64,3 & 4 & 28,6 & 1 & 7,1 & 14 & 100 \\
\hline Más de 10 años & 1 & 33,3 & 1 & 33,3 & 1 & 33,3 & 3 & 100 \\
\hline Total & 32 & 50 & 26 & 40,6 & 6 & 9,4 & 64 & 100 \\
\hline
\end{tabular}

Fuente: Elaboración propia con base en los datos recogidos en los cuestionarios realizados en Patio Bonito I, septiembre del 2013.

funcione y, específicamente, para la generación de compromisos que se estipulan de forma verbal y escrita, pero que en pocas situaciones alcanzan la forma del contrato registrado en notaría. Los compromisos son actualizados permanentemente e incluso pueden llevar a que formas escritas evolucionen hacia formas de palabra ya que la confianza prospera entre las partes, por ejemplo: cuando se pasa de una relación entre agentes económicos para dar paso a una relación de amistad, cuando surge un vínculo social además de uno económico.

\section{Conclusiones}

En Bogotá ha sido creciente el aumento de hogares que acceden a la vivienda a través del mercado de arrendamiento y, especialmente, de aquellos que cuentan 
Tabla 7. ¿ Para quién arrienda? Población arrendadora encuestada en Patio Bonito I

\begin{tabular}{|c|c|c|c|c|}
\hline & & Frecuencia & $\%$ & Acumulado \\
\hline \multirow{6}{*}{$\begin{array}{l}\text { Mayor grado de } \\
\text { personalización en la relación } \\
\text { con el arrendador }\end{array}$} & Solamente familiares & 5 & 6,8 & \multirow{6}{*}{41,9} \\
\hline & Solamente amigos & 12 & 16,2 & \\
\hline & Amigos y familiares & 2 & 2,7 & \\
\hline & Familiares y recomendados & 2 & 2,7 & \\
\hline & Amigos y recomendados & 8 & 10,8 & \\
\hline & Amigos, familiares y recomendados & 2 & 2,7 & \\
\hline \multirow{3}{*}{$\begin{array}{l}\text { Nivel intermedio de } \\
\text { personalización de la relación } \\
\text { con el arrendador }\end{array}$} & Recomendados y no recomendados & 9 & 12,2 & \multirow{3}{*}{52,8} \\
\hline & Amigos y no recomendado & 1 & 1,4 & \\
\hline & Persona desconocida pero recomendada & 29 & 39,2 & \\
\hline \multirow{2}{*}{$\begin{array}{l}\text { Predominio de relaciones } \\
\text { anónimas o con un mayor } \\
\text { grado de anonimato con el } \\
\text { arrendador }\end{array}$} & Persona desconocida sin recomendación & 4 & 5,4 & 5,4 \\
\hline & Total de observaciones & 74 & 100 & 100 \\
\hline
\end{tabular}

Fuente: Elaboración propia con base en los datos recopilados en los cuestionarios realizados en Patio Bonito I.

con los menores ingresos y que se localizan por lo general en barrios populares. Partiendo de una visión institucionalista del mercado se puede señalar que la distinción formal/informal muestra lo que se puede denominar pluralismo jurídico. En barrios como Patio Bonito I la regulación del mercado de arrendamiento involucra lo oficial como lo comunitario y deriva en un formalismo popular donde la trayectoria del barrio y su composición social son aspectos relevantes para comprender lo que desde una visión dicotómica se caracteriza como informalidad contractual.

El mercado de arrendamiento residencial está incrustado en lo social, pero

EL ARRENDAMIENTO RESIDENCIAL EN LOS BARRIOS POPULARES a la vez es generador de vínculos sociales. Como se pudo observar en el análisis de caso, los compromisos que los agentes económicos establecen pueden estar precedidos por la existencia de vínculos por parentesco, paisanaje o vecindad, pero existen relaciones contractuales que inician en un elevado grado de anonimato y que una vez alcanzada la confianza derivan en nuevos vínculos sociales. Gracias a la existencia de esas redes de relaciones sociales es que puede prosperar un sistema de regulación propio donde los compromisos se van ajustando hasta convertirse en convenciones. Quien no sigue esas convenciones puede ser objeto de sanción social e individual. territarios 38 
12 En entrevista realizada a un representante de la Oficina de Conciliación en Equidad se nos comentó que en barrios de origen formal también es frecuente observar conflictos entre arrendatarios $y$ arrendadores. La realización de acuerdos entre familiares y las dificultades para separar lo personal de lo estrictamente económico han llevado a que en varias ocasiones surjan problemas que envuelven a familiares.

\section{territarias 38} 114
Sin embargo, la ilusión de lo comunitario aparece en la medida en que se constata una baja participación en organizaciones de escala barrial, así como una cierta desconfianza hacia los demás vecinos, lo cual explicaría también por qué se requiere y se usa también la normatividad oficial.

Desde la perspectiva de la Escuela de Convenciones Francesa es posible un análisis que demuestra entonces la importancia de un examen interdisciplinar de las relaciones contractuales exento de un abordaje economicista. Desde la sociología se destaca que las relaciones económicas, y especialmente las contractuales, se caracterizan por su elevado grado de objetividad. No obstante, la existencia de este formalismo popular, de un pluralismo jurídico, nos lleva a plantear que los sistemas de regulación creados en los barrios populares apelan a la subjetivación de las relaciones económicas. Esta situación es importante para comprender la existencia o no de inseguridad en la tenencia de vivienda entre los arrendatarios de estos barrios informales.

El análisis de caso nos permite observar que dicha subjetivación facilita la celebración de los compromisos, pero también señala la posibilidad de crear mecanismos propios para la resolución de los conflictos que puedan surgir entre las partes, sin que se tenga que apelar a las instancias jurídicas oficiales. Se trata de una justicia alternativa que en Bogotá ha recibido un impulso desde el mismo Estado en las últimas dos décadas, por la vía de 'conciliadores en equidad' o de 'jueces de paz'. No obstante, cuando las partes no pueden conciliar por su cuenta o a través de una instancia comunitaria tienen lugar situaciones de resolución que apelan a la violencia.

Una excesiva subjetivación de las relaciones contractuales lleva efectivamente a una indefinición de los derechos de propiedad y, por ende, a que los conflictos tiendan a darse incluso entre parientes. En estos casos la terminación violenta de la relación contractual no solo acaba con una relación económica, sino que afecta los vínculos sociales que pueden germinar en este tipo de barrios $^{12}$. Si bien se puede cuestionar hasta dónde es bueno hacer negocios con amigos o familiares, en el caso de mercados como el de arrendamiento residencial es claro también que de relaciones exclusivamente económicas es posible que surjan nuevos vínculos sociales.

Las familias de bajos ingresos recurren a estas redes con el propósito de acceder a una vivienda que sea económica y próxima de sus familiares. Si bien la existencia de las redes de relaciones sugiere la posibilidad de que surjan valores como la solidaridad y la cooperación, también pueden señalar que en ellas se está rehén de jerarquías, de relaciones de autoridad que pueden derivar en situaciones de privación que requieren de intervención por la vía de políticas públicas. Así pues, desde el Estado, e incluso desde las mismas organizaciones o movimientos por la vivienda, será importante comprender estos sistemas de regulación populares para aprovecharlos en pro de la generación de iniciativas de vivienda social en alquiler. 


\section{Referencias}

Abramo, P. (2002). Uma teoria econômica da favela: quatro notas sobre o mercado imobiliário informal em favelas e a mobilidade residencial dos pobres. Cadernos IPPUR, XVI(2), 103-134.

Abramo, P. (2009). A cidade informal COMFUSA: mercado informal em favelas e a produção da estrutura urbana nas grandes metrópoles latino-americanas. En P. Abramo (org.). Favela e mercado informal: a nova porta de entrada dos pobres nas cidades brasileiras (vol. 10, pp. 49-79). Porto Alegre: ANTAC, Coleção Habitare.

Bejarano, J. (1999). El análisis económico del derecho: comentarios sobre textos básicos. Revista Economia Institucional, l(1), 155-167. Recuperado de http:// www.economiainstitucional.com/pdf/ Nol/jbejaranol.pdf

Blanco, A., Fretes, V. \& Muñoz, A. (2014). Se busca vivienda en alquiler: opciones de politica en América Latina y el Caribe. Washington: Banco Interamericano de Desarrollo - BID.

Blanco, A. \& Volpe, F. (2015). Alquiler en números: la tenencia de vivienda en América Latina y el Caribe. Washington: Banco Interamericano de Desarrollo - BID. Recuperado de https://publications.iadb.org/handle/11319/6888

Bowles, S. (2004). Microeconomics: behavior, institutions and evolution. New York: Rusell Sage.

Briceño-León, R. (2010). Diez tesis sobre los pobres y el alquiler de viviendas. En:
C. Escallón (comp.), Arrendamiento y Vivienda Popular en Colombia como alternativa Habitacional: Mesa VIS Diego Echeverry Campos (pp. 105-124). Bogotá: Secretaría Distrital del Hábitat y Universidad de Los Andes. Ediciones Uniandes.

Coase, R. (1994). El problema del costo social. En F. Aguilera \& V. Alcántara (orgs.). De la economía ambiental a la economía ecológica (pp. 41-77). Barcelona: Fuhem e Icaria. Recuperado de http://www.fuhem.es/media/ecosocial/File/Actualidad/2011/Coase.pdf Colombia. Congreso de la República. Ley 820 de 10 de julio de 2003. Reglamentación sobre arrendamiento residencial urbano. Recuperado de http://www. alcaldiabogota.gov.co/sisjur/normas/ Normal.jsp? $\mathrm{i}=8738$

Díaz, M. (2003). El nacimiento y constitución del barrio Patio Bonito y la importancia de la Junta de Acción Comunal en su desarrollo. Una mirada comunicacional. 2003. 244 f. (Tesis de pregrado en comunicación social, Facultad de ciencias sociales y humanas, Universidad Nacional Abierta y a Distancia - UNAD, Bogotá).

Eymard-Duvernay, F. (2001). Economía de las convenciones y su aplicación al estudio de las empresas y los mercados. Buenos Aires: Programa de investigaciones económicas sobre tecnología, trabajo y empleo - PIETTE. Recuperado de http:// www.ceil-conicet.gov.ar/wp-content/ uploads/2013/06/s8eymard.pdf territarias 38

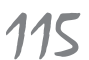

EL ARRENDAMIENTO RESIDENCIAL EN LOS BARRIOS POPULARES 
Favereau, O. (1993). Teoría de la regulación y economía de las convenciones: boceto para una confrontación. Noticias de la regulación, 7, 1-5. Recuperado de http://www.ceil-conicet.gov.ar/publicaciones/noticias-de-la-regulacion/

Fiani, R. (2002). Teoria dos custos de transação. En D. Kupfer \& L. Hasenclever (orgs.). Economia industrial. Fundamentos teóricos e práticas no Brasil. Rio de Janeiro: Elsevier.

Fiani, R. (2011). Cooperação e conflito. Instituições e desenvolvimento econômico. Rio de Janeiro: Elsevier.

Gilbert, A. (1993). In search of a home: rental and shared housing in Latin America. London: UCL Press.

Granovetter, M. (1985). Economic action and social structure: the problem of embeddedness. American Journal of Sociology, 91(3), 481-510. Recuperado de http://www.jstor.org/discover/10 $.2307 / 2780199$ ? $\mathrm{sid}=2110513071106$ 1 \&uid $=2129$ \&uid $=4 \&$ uid $=3737664 \&$ uid $=70 \&$ uid $=2134 \&$ uid $=2$

Hataya, N. (2009). La ilusión de la participación comunitaria: lucha y negociación en los barrios irregulares de Bogotá 19922003. Bogotá: Universidad Externado de Colombia.

Hirschman, A. (1977). Salida, voz y lealtad. Respuestas al deterioro de empresas, organizaciones y estados. México: Fondo de Cultura Económica.

Lacerda, N. (2011). Mercado imobiliário de aluguel em áreas pobres e teoria das

\section{territarias 38} convenções. Recife: Centro de Estudos
Avançados da conservação integrada CECI.

Lacerda, N., Paulo, A., Cruz, D. \& Dos Anjos, K. (2010). Mercado imobiliário de aluguel em áreas pobres: normas de funcionamento e sentidos da informalidade. En S. Leal \& N. Lacerda (orgs.). Novos padrões de acumulação urbana na produção urbana do habitat: olhares cruzados Brasil-França (pp. 241-269). Recife: Ed. Universitária UFPE.

Magalhães, A. (2013). Sociologia do Direito. Opluralismo jurídico em Boaventura de Sousa Santos. Rio de Janeiro: Editora da Universidade Federal do Rio de Janeiro.

North, D. (1999). Understanding the process of economic change. Londres: Institute of economic affairs.

Organización de las Naciones Unidas - ONU (2012). Resolución A/HRC/22/46 del 24 de diciembre. Recuperado de http:// direitoamoradia.org/wp-content/ uploads/2013/02/A.HRC_.22.46_ sp.pdf

Parias, A. (2008). El mercado de arrendamiento en los barrios informales en Bogotá, un mercado estructural. Territorios, (18-19), 75-101. Recuperado de http://revistas.urosario.edu.co/index. php/territorios/article/view/828

Parias, A. \& Abramo, P. (2009). Mercado informal de suelo en los barrios populares de Bogotá: claves para entender el crecimiento de la metrópolis. En P. Abramo (org.). Favela e mercado informal: a nova porta de entrada dos pobres nas cidades brasileiras (vol. 10, pp. 305- 
336). Porto Alegre: ANTAC, Coleção Habitare.

Sáenz, H. (2009). La práctica del arrendamiento habitacional en cuatro barrios populares de Bogotá. Revista Otra Economia, 3(4), 155-173. Recuperado de http://revistas.unisinos.br/index. $\mathrm{php} /$ otraeconomia/article/view/1130

Sáenz, H. (2016). La subjetivación de las relaciones económicas. Reflexiones para una tipología de mercados informales de arrendamiento residencial. Polis Revista Latinoamericana, 15(45), 235-248. Recuperado de http:// www.scielo.cl/scielo.php?pid=S0718$65682016000300012 \&$ script $=$ sci arttext\&tlng=en

Secretaría Distrital de Hábitat - SDH (2014). Abecé del vendedor y arrendador de vivienda y los derechos del ciudadano. Bogotá: Secretaría Distrital de Hábitat. Recuperado de http://www.habitatbogota.gov.co/sdht/images/documentos/cartilla_arrendadores_enajenadores.pdf
Secretaría Distrital de Planeación - SDP (2015). Encuesta multipropósito 2014. Principales resultados en Bogotá y la región. Bogotá: Serie Bogotá, ciudad de estadísticas, Boletín 65 .

Torres, J. (2012). Estudio sobre el mercado de arrendamiento de vivienda en Colombia. Bogotá: Banco Interamericano de Desarrollo - BID. Recuperado de http:// www.cenac.org.co/

Torres, J. \& Pérez, E. (2008). Caracterización del mercado de arrendamiento urbano para el segmento de la población de bajos ingresos. Revista INVI, 23(63), pp. 53-87. Recuperado de http://revistainvi.uchile.cl/index. $\mathrm{php} / \mathrm{INVI} /$ article/view/443

Williamson, O. (1986). Economic organization: firms, markets and political control. New York: New York University Press. Williamson, O. (1996). The mechanism of governance. Oxford: Oxford University Press.

Younkis, E. (2000). Freedom to contract. Quebec: Liberty Free Press. Recuperado de http://www.quebecoislibre. org/younkins $25 . h t m l$ 
